http://dx.doi.org/10.18778/2196-8403.2015.10

ANNA DASZKIEWICZ

\title{
Zur gegenwärtigen Diskussion über die ethnolek- talen Merkmale des Jugend-Deutschen
}

Niniejszy tekst ma na celu rekonstrukcję wiedzy zgromadzonej na temat etnolektu (etnicznej odmiany języka napływowej i natywnej młodzieży mieszkającej w Niemczech) w świetle publikacji medialnych, tj. felietonów, esejów, reportaży i wywiadów. Relacje medialne podejmują próby scharakteryzowania etnolektu poprzez odniesienia do bardziej aktualnych dostępnych na rynku publikacji językoznawców i ekspertów.

Der vorliegende Text hat die Rekonstruktion des Wissens um den Ethnolekt (die ethnisch geprägte Sprachvarietät Jugendlicher deutscher und fremdstämmiger Jugend) anhand der medial aufbereiteten und popularisierten Berichterstattung, d.h. Feuilletons, Essays, Berichte und Interviews zum Ziel. Durch den Zugriff auf aktuelle und auf dem Markt erhältliche Veröffentlichungen anerkannter Sprachwissenschaftler und Experten wird im medialen Rahmen die Charakterisierung des besagten Sprachphänomens ermöglicht.

This text is meant to reconstruct the state of knowledge gathered thus far on the issue of ethnolect (ethnic language variant of the incoming and native youth residing in Germany) in the light of media publications, that is columns, essays, reports and interviews. Media reports undertake attempts to characterise ethnolect by references to the more valid publications of linguists and experts in this discipline available on the market.

\section{Einleitung}

Ziel des vorliegenden Beitrags ist, den bisherigen Wissensstand über die Tendenzen des gesprochenen Deutsch durch das Prisma der aktuellen Berichterstattung aufzuzeigen und dafür zu sensibilisieren. Eine enorme Medienresonanz erfährt in den letzten Jahrzehnten die deutsch-türkische Mischvariante, 
die der Gegenstand und mitunter auch ein ,Dorn im Auge“ der modernen Linguistik sowie linguistisch orientierten Publizistik ist. Die Ergebnisse der bundesweit durchgeführten Feldforschungsprojekte zu Kreuzungs- und Mischungsprozessen im Umfeld der deutsch- und andersstämmigen Jugend münden in zahlreiche und interesseheischend wirkende Publikationen. Diese werden im medialen Rahmen aufgegriffen und kommentiert. Eben diesen medial vermittelten Kommentaren (Besprechungen des bereits Erforschten und Dokumentierten) wird in diesem Beitrag Rechnung getragen.

Hierbei sei erwähnt und betont, dass anerkannte und zu diesem Thema forschende Linguisten mitunter Feuilletons- / Essays-Verfasser sind, so dass deren Gesamtwerk als Mitkonstituente des allgemeinen Medienwissens fungiert. Kurzum werden Fallstudienergebnisse und deren Autoren selbst zur Zielscheibe der Kritik und des medialen Interesses.

Damit der Wissensstand über den ethnisch geprägten Sprechstil deutschsprachiger Jugendlicher anhand von aktuellen Medienberichten rekonstruiert werden kann, ist zunächst die Beleuchtung und Besprechung dieses Sprachphänomens aus sprachwissenschaftlicher Warte (als theoretische Grundlage) erforderlich. Diese wird anschließend um die mediale Berichterstattung dazu ergänzt und so bereichert.

\section{Der ,multiethnische Sprech“}

Wie bereits eingangs angedeutet, wird in letzter Zeit auf einen ethnisch geprägten Sprechstil Jugendlicher in der Bundesrepublik ein besonderes Augenmerk gelegt. Aufgrund vieler Kontroversen in Bezug auf den angeblich damit zusammenhängenden Sprachwandel ist das ,Kauderwelsch“ medialer Inhalt geworden. Hierbei ist mit dem ,multiethnischen Sprech`,eine Art sozial-kommunikativer Sprechstil' gemeint, der von fremdstämmigen und deutschen Jugendlichen in multiethnischen Wohngebieten dermaßen erfolgreich praktiziert wird, dass er inzwischen eine in sich stimmige Morphosyntax aufweist. Hierbei sind die systematischen Neuerungen in Grammatik, Wortschatz und Aussprache folgende:

So wird aus ,ich“ beispielsweise „isch“, was ähnlich im Rheinland vorkommt und im Berliner „nüscht“. Wir finden neue Funktionswörter wie „lassma“ und „musstu“ („lass uns mal“ und „musst du“) und Zusammenziehungen wie „ischwör“ (,ich schwöre“), mit dem eine Aussage bekräftigt wird - ganz ähnlich, wie umgangssprachlich die Zusammenziehung „glaubich“ (,glaube ich“) eine Aussage abschwächt. Das Wort „so“ wird nicht nur zum Vergleich verwendet, sondern 
auch zur Betonung („Ich höre Alpa Gun, weil er so aus Schöneberg kommt.“), so entsteht ein neues Funktionswort, das wir übrigens auch außerhalb von Kiezdeutsch finden. Das ist nicht schlampig formuliert, sondern hat System. Im Ethnolekt schrumpft der deutsche Gesamtwortschatz kontinuierlich zusammen. Einfache Satzkonstruktionen werden aus Subjekt, Prädikat und Objekt gebildet, Artikelformen und Präpositionen weggelassen (,wenn wir Hochzeit gehen“), Genera verändert („,son großer Plakat“), und oft fehlen die Pronomen („die haben mir beigebracht“). Beim sogenannten Codeswitching werden mitten im Satz türkische, arabische oder serbokroatische Lehnwörter eingebunden: „yalla“ für „,auf geht’s“ oder „wallah“ (bei Gott), wenn etwas mit Nachdruck versichert wird. „Isch schwör“ bekräftigt eine Aussage, alles in Ordnung heißt „Tamam“, und Einschübe wie „Lan“ oder „Moruk“ sind im Ethnolekt ebenso geläufig wie die deutsche Entsprechung: „Alda“. Außerdem zählen Ausrufe wie „weissu“, „krass“ oder „korrekt“ dazu. Wichtig bei der Aussprache ist die spezielle Stakkato-Intonation - und typisch die sogenannte Koronalisierung des Ich-Lauts: Isch, misch, disch. Ein mustergültiger Satz lautet: „Isch geh gleisch U-Bahn. (HARUNA 2011:1)

Aufgrund zahlreicher und merklicher Abweichungen dem Standarddeutschen gegenüber wird der ethnolektale ${ }^{1}$ Sprechstil im öffentlichen Diskurs meist abwertend, und zwar als „eine verkürzte, abgehackte Sprak“ (HARUNA 2011:1), „ein eigenartiges nicht Duden-kompatibles Gossen-Stakkato“ (WIESE 2012:1), „ein reduziertes Deutsch ohne Grammatik“ (WIESE 2012:1), „ein abschwellender Straßenslang“ (BAX 2001:1), „Kauderwelsch“ (BRAUN 2000:1) oder „Sprachmischmasch“ (KRUMMHEUER 2007:1), dessen Vokabular „einer Notrotation gleicht“ (WIESE 2012:1), dargestellt. Für ein derartiges evaluatives Deutungsmuster sorgen Medienbeiträge selbst, die konsequent mit griffigen und prägnanten Titelbezeichnungen des Typs „Lass ma’ lesen, yallah!“ (TWIEHAUS 2012:1), „Ich bin Alexanderplatz“ (SERRAO 2012:1), „Ich geh Schule“ (KRISCHKE 2006:1), „Hab isch gesehen mein Kumpel“ (HINRICHS 2012:104), „Ischwör, morgen bin ich Arzt!“ (KRISCHKE 2016:1), „,Gehst du Bus?‘ Kiezdeutsch gibt's bald ohne Kiez“ (von LESZCZYNSKi 2014:1) arbeiten und den besagten Sachverhalt, tendenziell (klischeehaft sowie karikierend) vermittelnd, ins Blickfeld einer breiteren Leserschaft bringen. Der besseren Übersichtlichkeit und Verständlichkeit halber werden an dieser Stelle einige aufmerksamkeitsheischende Titel und Untertitel von aktuellen Medienbeiträgen, die das besagte Sprachphänomen hematisieren, aufgelistet und in Form einer Tabelle dargestellt:

Unter dem Fachbegriff Ethnolekt verstehen PETER AuER und ǏNCI DiRIM eine Sprechweise, die von ethnisch und jugendlich geprägten Gruppen verwendet und als für sie charakteristisch wahrgenommen wird (AUER 2003: 255-264, DIRIM / AUER 2004: 218, 220-223). 
Tabelle 1

\begin{tabular}{|c|c|c|c|}
\hline & Titel des Beitrags & Untertitel des Beitrags & Quelle/Autor/Datum \\
\hline 1. & $\begin{array}{l}\text { „Kanak Sprak“: } \\
\text { Verhunztes } \\
\text { Deutsch oder } \\
\text { Sprachkultur? }\end{array}$ & $\begin{array}{l}\text { Wächter des Hochdeutschen wittern } \\
\text { Sprachverfall - Sprachforscher di- } \\
\text { agnostizieren kreative Sprachvielfalt } \\
\text { und sagen: Jugendsprachen bedro- } \\
\text { hen die deutsche Sprache nicht, sie } \\
\text { bereichern sie. Doch wie kreativ ist } \\
\text { der „multi-ethnische Sprech“ tat- } \\
\text { sächlich? }\end{array}$ & $\begin{array}{l}\text { http://www.br.de/ra- } \\
\text { dio/bayern2/wissen/iq- } \\
\text { wissenschaft-und-for- } \\
\text { schung/gesell- } \\
\text { schaft/Sprachver- } \\
\text { fall100.html. } \\
\text { Von MARTIN SCHRAMM, } \\
\text { veröffentlicht am } \\
\text { 31.05.2011. }\end{array}$ \\
\hline 2. & $\begin{array}{l}\text { Sprache: } \\
\text { Zukunftssprache } \\
\text { Kanak Sprak? }\end{array}$ & $\begin{array}{l}\text { Die Einwanderung verändert auch } \\
\text { die deutsche Sprache. Manche Lin- } \\
\text { guisten erwarten gar eine radikale } \\
\text { Vereinfachung der Grammatik - } \\
\text { auch in der Schriftsprache. }\end{array}$ & $\begin{array}{l}\text { http://www.dw.com/de/z } \\
\text { ukunftssprache-kanak- } \\
\text { sprak/a-15208590. } \\
\text { Von DENNIS STUTE, ver- } \\
\text { öffentlicht am } \\
\text { 04.07.2011. } \\
\end{array}$ \\
\hline 3. & $\begin{array}{l}\text { Weissu - is krasse } \\
\text { Sprache! } \\
\text { Jugendliche Mig- } \\
\text { ranten mischen } \\
\text { das Hochdeutsch } \\
\text { auf }\end{array}$ & $\begin{array}{l}\text { In der Clique, auf dem Schulhof, im } \\
\text { Viertel: Überall kann eine eigene } \\
\text { Sprache entstehen. Gerade Jugendli- } \\
\text { che verändern das Hochdeutsch krea- } \\
\text { tiv, um sich abzugrenzen, oder ein- } \\
\text { fach aus Spaß an der Sprache. Ein } \\
\text { Lauschangriff. }\end{array}$ & $\begin{array}{l}\text { http://www.flu- } \\
\text { ter.de/de/spra- } \\
\text { chen/heft/9536/. } \\
\text { Von HADIJA HARUNA, } \\
\text { veröffentlicht am } \\
\text { 30.08.2011. }\end{array}$ \\
\hline 4. & $\begin{array}{l}\text { Jugendsprache: } \\
\text { Lass ma' lesen, } \\
\text { yallah! }\end{array}$ & $\begin{array}{l}\text { Bei Kiezdeutsch gehen Sprachbe- } \\
\text { wahrer die Wände hoch. Sie bangen } \\
\text { um die Reinheit der Sprache Goe- } \\
\text { thes oder Schillers. Weil sie den } \\
\text { Schulhof-Slang für wertvoll hält, } \\
\text { wird Sprachforscherin Heike Wiese } \\
\text { regelmäßig angefeindet. Sie findet: } \\
\text { Die Jugendsprache ist oft viel logi- } \\
\text { scher als Standarddeutsch. }\end{array}$ & $\begin{array}{l}\text { http://www.spie- } \\
\text { gel.de/schulspiegel/le- } \\
\text { ben/jugendsprache-lass- } \\
\text { ma-lesen-yallah-a- } \\
\text { 811877.html. } \\
\text { Veröffentlicht am } \\
\text { 28.01.2012. }\end{array}$ \\
\hline 5. & $\begin{array}{l}\text { Jugenddialekt } \\
\text { Kiezdeutsch: „Ich } \\
\text { bin Alexander- } \\
\text { platz“ }\end{array}$ & $\begin{array}{l}\text { Unvollständige Sätze, durchmischt } \\
\text { mit arabischen und türkischen Wor- } \\
\text { ten: Kiezdeutsch, die Jugendsprache } \\
\text { der Großstädte, hat keinen guten } \\
\text { Ruf. Zu Unrecht, sagt Sprachwissen- } \\
\text { schaftlerin Heike Wiese: Der Slang } \\
\text { wird als Dialekt des Deutschen er- } \\
\text { halten bleiben. }\end{array}$ & $\begin{array}{l}\text { http://www.sueddeut- } \\
\text { sche.de/leben/jugenddi- } \\
\text { alekt-kiezdeutsch-ich- } \\
\text { bin-alexanderplatz- } \\
\text { 1.1278128. } \\
\text { Von MARC FELIX SER- } \\
\text { RAO, veröffentlicht am } \\
08.02 .2012 .\end{array}$ \\
\hline 6. & $\begin{array}{l}\text { Essay: Hab isch } \\
\text { gesehen mein } \\
\text { Kumpel }\end{array}$ & $\begin{array}{l}\text { Wie die Migration die deutsche } \\
\text { Sprache verändert hat }\end{array}$ & $\begin{array}{l}\text { http://www.spie- } \\
\text { gel.de/spiegel/print/d- } \\
\text { 83977255.html. } \\
\text { Von UWE HiNRICHS, } \\
\text { veröffentlicht am } \\
\text { 13.02.2012. }\end{array}$ \\
\hline
\end{tabular}


Tabelle 1, Fortsetzung

\begin{tabular}{|c|c|c|c|}
\hline 7. & $\begin{array}{l}\text { „Das Deutsch } \\
\text { der Migranten } \\
\text { gibt es nicht.“ } \\
\text { Die Soziolinguis- } \\
\text { tin Inken Keim } \\
\text { über Standard- } \\
\text { und „Kiez- } \\
\text { deutsch“ }\end{array}$ & $\begin{array}{l}\text { Viele Bürger mit Migrationshinter- } \\
\text { grund sprechen kein reines Hoch- } \\
\text { deutsch. Besonders Jugendliche un- } \\
\text { terhalten sich in einem eigenen, nach } \\
\text { den Sprachregeln völlig falschem, } \\
\text { Slang. Kein Problem, sagt die Sozio- } \\
\text { linguistin Inken Keim - solange die } \\
\text { Kinder auch Standarddeutsch lernen, } \\
\text { wenn sie später eine Berufsausbil- } \\
\text { dung machen oder studieren wollen. }\end{array}$ & $\begin{array}{l}\text { http://www.deutschland- } \\
\text { funk.de/das-deutsch-der- } \\
\text { migranten-gibt-es- } \\
\text { nicht.691.de.html?dram: } \\
\text { article_id=56817. } \\
\text { Das Gespräch führte } \\
\text { STEFAN KOLDEHOFF, } \\
\text { veröffentlicht am } \\
\text { 13.03.2012. }\end{array}$ \\
\hline 8. & $\begin{array}{l}\text { Plädoyer einer } \\
\text { Professorin: } \\
\text { Kiezdeutsch } \\
\text { rockt, ischwör! }\end{array}$ & $\begin{array}{l}\text { Sprachbewahrer kämpfen verbissen } \\
\text { gegen Kiezdeutsch. Der Schulhof- } \\
\text { Slang verhunzt unsere Sprache, mei- } \\
\text { nen sie. Alles Quatsch, sagt die } \\
\text { Sprachforscherin Heike Wiese. Denn } \\
\text { Kiezdeutsch sei genauso ein Dialekt } \\
\text { wie Bayerisch und Schwäbisch. Ein } \\
\text { Gastbeitrag. }\end{array}$ & $\begin{array}{l}\text { http://www.spie- } \\
\text { gel.de/unispiegel/wun- } \\
\text { derbar/professorin-heike- } \\
\text { wiese-verteidigt-den-ju- } \\
\text { gendslang-kiezdeutsch-a- } \\
\text { 824386.html. } \\
\text { Von HEIKE WIESE, ver- } \\
\text { öffentlicht am } \\
\text { 29.03.2012. } \\
9\end{array}$ \\
\hline 9. & $\begin{array}{l}\text { Sprache im Wan- } \\
\text { del: } \\
\text { Präposition } \\
\text { überbewertet }\end{array}$ & $\begin{array}{l}\text { An manchen Schulen sind Sätze wie } \\
\text { „Gehst du Bus?“ oder „Hast du Lo- } \\
\text { cher?“ längst gang und gäbe. In der } \\
\text { Zukunft könnten alle Menschen in } \\
\text { Deutschland so reden, sagen Sprach- } \\
\text { forscher. }\end{array}$ & $\begin{array}{l}\text { http://www.faz.net/aktu- } \\
\text { ell/gesellschaft/sprache- } \\
\text { im-wandel-praeposition- } \\
\text { ueberbewertet- } \\
\text { 13016742.html. } \\
\text { Von HEIKE LINKO, ver- } \\
\text { öffentlicht am } \\
\text { 29.06.2014. }\end{array}$ \\
\hline 10. & $\begin{array}{l}\text { Sprachwandel } \\
\text { „Gehst du Bus?“ } \\
\text { Kiezdeutsch } \\
\text { gibt's bald ohne } \\
\text { Kiez }\end{array}$ & $\begin{array}{l}\text { „Kommst du mit Klo?“ oder „Ich war } \\
\text { Fußball“ - solche Sätze sind an man- } \\
\text { chen Berliner Schulen Alltag. Eine } \\
\text { neue Umgangssprache entsteht, sa- } \\
\text { gen Sprachforscher und glauben: } \\
\text { Bald könnten wir alle so reden. }\end{array}$ & $\begin{array}{l}\text { http://www.welt.de/ver- } \\
\text { mischtes/ar- } \\
\text { ticle129589372/Gehst- } \\
\text { du-Bus-Kiezdeutsch- } \\
\text { gibts-bald-ohne- } \\
\text { Kiez.html. } \\
\text { Von ULRIKE von LESZ- } \\
\text { CZYNSKI, veröffentlicht } \\
\text { am 29.06.2014. } \\
\end{array}$ \\
\hline 11. & $\begin{array}{l}\text { Streit um Spra- } \\
\text { che: In Wahrheit } \\
\text { ist Kiezdeutsch } \\
\text { rassistisch }\end{array}$ & $\begin{array}{l}\text { Zwei Germanistinnen versuchen uns } \\
\text { einzureden, Kiezdeutsch sei der Stan- } \\
\text { dardsprache gleichrangig und jeder, } \\
\text { der auf korrektem Deutsch beharre, } \\
\text { sei ein Rassist. Türkische Aufsteiger } \\
\text { wissen es besser. }\end{array}$ & $\begin{array}{l}\text { http://www.welt.de/kul- } \\
\text { tur/article129622721/In- } \\
\text { Wahrheit-ist-Kiez- } \\
\text { deutsch-rassistisch.html. } \\
\text { Von MATTHIAS HEINE, } \\
\text { veröffentlicht am } \\
\text { 30.06.2014. }\end{array}$ \\
\hline
\end{tabular}


Tabelle 1, Fortsetzung

\begin{tabular}{|c|c|c|c|}
\hline 12. & $\begin{array}{l}\text { Kurzdeutsch: } \\
\text { „Ich gehe Dö- } \\
\text { ner“ ist längst im } \\
\text { Sprachgebrauch } \\
\text { angekommen }\end{array}$ & $\begin{array}{l}\text { Kiezdeutsch heißt jetzt Kurzdeutsch. } \\
\text { Eine Linguistin erklärt, warum Ju- } \\
\text { gendliche Sätze wie „Verstehst du } \\
\text { Text, Mann?“ oder „Ich gehe Döner“ } \\
\text { sagen. Längst reden nicht mehr nur } \\
\text { Migrantenkinder so. }\end{array}$ & $\begin{array}{l}\text { http://www.welt.de/kul- } \\
\text { tur/literarischewelt/ar- } \\
\text { ticle151650918/Ich- } \\
\text { gehe-Doener-ist-laengst- } \\
\text { im-Sprachgebrauch-an- } \\
\text { gekommen.html. } \\
\text { Von MATTHIAS HEINE, } \\
\text { veröffentlicht am } \\
\text { 30.01.2016. }\end{array}$ \\
\hline 13. & $\begin{array}{l}\text { Kiezdeutsch: Is- } \\
\text { chwör, morgen } \\
\text { bin ich Arzt! }\end{array}$ & $\begin{array}{l}\text { Wenn Minimalgrammatik zur } \\
\text { Sprachreform verklärt wird: Die So- } \\
\text { ziolinguistin Diana Marossek traut } \\
\text { dem Kiezdeutschen einiges zu. Ihre } \\
\text { Untersuchung überzeugt, ihre Prog- } \\
\text { nose nicht. }\end{array}$ & $\begin{array}{l}\text { http://www.faz.net/aktu- } \\
\text { ell/feuilleton/buecher/re- } \\
\text { zensionen/sach- } \\
\text { buch/kiezdeutsch-unter- } \\
\text { sucht-von- } \\
\text { soziolinguistin-diana- } \\
\text { marossek- } \\
\text { 14167926.html. } \\
\text { Von WOLFGANG } \\
\text { KRISCHKE, veröffentlicht } \\
\text { am 14.04.2016. }\end{array}$ \\
\hline
\end{tabular}

Des Weiteren kommt die ethnolektale Mischvariante in vielfältiger Ausprägung zum Vorschein: Ihrem Namen haften lexemspezifische, konnotative Unterschiede (ethnie- oder ortübergreifende Erstglieder) an. Demnach wird dieses Sprachphänomen als ,Kanak Sprak‘, ,Türkendeutsch‘, ,Türkenslang‘, ,Dönerdeutsch“, ,Kebabdeutsch“, ,Lan-Sprache‘, ,Kiezsprache‘, ,Kiezdeutsch“, ,Ghettodeutsch` oder ,Balkandeutsch` bezeichnet und ikonisiert, was zum einen auf seinen ,einzelethnolektalen', zum anderen ,multiethnolektalen` Charakter und seine Verankerung im deutschen Sprachsystem im Sinne von CLYNE (2000) hindeutet.

Es sei hier aber ergänzend hinzugefügt, dass die medial ausgedrückte Sorge um die Zukunft des Standarddeutschen (sprich: das Lamento) durch eine umgekehrte Sichtweise, die diesen einflussreichen Sprachtrend als eine kreative Innovation und Bereicherung empfinden lässt, ausbalanciert werden will. Demgemäß wird auf die Mischsprache Jugendlicher mithilfe eines binären Oppositionsmusters: „verhunztes Deutsch oder Sprachkultur“ (SCHRAMM 2011:1, Hervorhebung A.D.), „Sprachverfall oder sprachlicher Spezialfall“ (CANOĞLU 2012, Hervorhebung A.D.), „kommunikative Einschränkung oder sprachökonomische Reform“ (KRISCHKE 2016:1, Hervorhebung A.D.), „kreativ oder naiv“ (SCHRAMm 2011:1, Hervorhebung A.D.) fokussiert. Die Auffassung des ethnolektalen Sprechstils nach dem binären Oppositionsmodell schweißt sogar weit auseinander liegende Standpunkte (konträre Stimmen von 
Wächtern des Hochdeutschen und der ethnolektalen Sprechart verschriebenen Linguisten) zusammen, die allesamt eine damit einhergehende dauerhafte Veränderung des gesprochenen Deutsch wittern:

Es verändert sich vor allem das gesprochene Deutsch, also die Umgangssprache, weil die alltägliche Mehrsprachigkeit fast ausschließlich mündlich auftritt. Das offiziöse Schriftdeutsch ist träge und spiegelt von den neuesten Veränderungsprozessen nur wenig wider (allenfalls in spontanen Fehlern), und so vergrößert sich die Distanz zwischen geschriebenem und gesprochenem Deutsch rapide. (HINRICHS 2012:104)

Einer genaueren Erforschung der ethnisch geprägten Sprachvariante haben sich bisher drei deutsche Universitäten verschrieben und zu diesem Zweck entsprechende Projekte (das Potsdamer, das Mannheimer und das Stuttgarter Projekt) erstellt. Hierbei stellen in den drei Fällen authentische Korpora von der In-Group-Kommunikation Jugendlicher im Alter zwischen 12 und 22 Jahren (der transkribierte Datensatz) die Grundlage zur linguistischen Auswertung. Obwohl die Projektteilnehmer aus unterschiedlichen Forschungsrichtungen und mit unterschiedlichen Methoden an sprachbezogene Mixing-Strategien Jugendlicher herangehen, steht im Vordergrund ihrer Betrachtung die Untersuchung der besagten Mischvariante in Richtung eines selbständigen, in sich stimmigen Sprechstils oder ganz im Gegensatz dazu, einer Übernahme der fossilisierten Zweitspracherwerbsstufe des Deutsch ehemaliger ,Gastarbeiter". Sprachliche Besonderheiten werden jeweils in phonetisch-phonologischer, morphologisch-syntaktischer und lexikalischer Hinsicht und bezogen auf ihre Funktionen analysiert und charakterisiert. Der besseren Nachvollziehbarkeit halber werden hiermit die Grundthesen und Ergebnisse der im Rahmen der Projekte durchgeführten Fallstudien in Form einer Tabelle veranschaulicht:

Tabelle 2

\begin{tabular}{|l|l|l|}
\hline & Bezeichnung des Projekts & Hauptanliegen \\
\hline 1. & Das Potsdamer Projekt & Dieses Projekt arbeitet unter dem Motto „Türkendeutsch \\
& & in interaktionallinguistischer Perspektive“ und sieht in \\
& der besagten Varietät einen „neuen, ethnischen Sprech- \\
& und Interaktionsstil des Deutschen“ (KERN / SELTING \\
& 2006:240). Durch intensive Feldforschungen wurde hier \\
& nachgewiesen, dass die ethnische Mischvariante über \\
& eine eigene, gut ausgebaute Morphosyntax verfügt - \\
& demnach als ein neuer Dialekt des Deutschen anerkannt \\
& (vgl. WIESE 2012) und mit dem defizitären ,Gastarbei- \\
& terdeutsch` der ersten Migrantengeneration nicht mehr \\
& gleichzusetzen ist. \\
\hline
\end{tabular}


Tabelle 2, Fortsetzung

\begin{tabular}{|c|c|c|}
\hline 2. & Das Mannheimer Projekt & $\begin{array}{l}\text { Dieses Projekt wird vom Institut für Deutsche Sprache } \\
\text { durchgeführt und trägt den Titel „Deutsch-Türkische } \\
\text { Sprachvariation und die Herausbildung kommunikativer } \\
\text { Stile in jugendlichen Migrantengruppen in Mannheim“. } \\
\text { Das Konzept des kommunikativen Sozialstils wurde von } \\
\text { WERNER KALLMEYER und seinen Mitarbeitern im Laufe } \\
\text { der 1990er Jahre entwickelt. Wichtigste Veröffentlichun- } \\
\text { gen dazu sind folgende: KALLMEYER (1994), KALLMEYER } \\
\text { (1995), KEIM (1995) und ScHWITALLA (1995). Mit dem } \\
\text { Konzept der kommunikativen Sozialstile wird „die Art und } \\
\text { Weise der Kommunikation, die mit gewissen sozialen Ei- } \\
\text { genschaften verbunden und in der Regel mit bestimmten } \\
\text { sozialen Kategorien benannt wird“ (CINDARK 2005:300), } \\
\text { erfasst. Da der kommunikative Stil Identitätssymbole } \\
\text { transportiert (identitätsstiftend wirkt), ist ihm eine soziale } \\
\text { Dimension zuzuschreiben. } \\
\text { Dieser Stilbegriff steht im Zusammenhang mit dem For- } \\
\text { schungsansatz der „Ethnographie der Kommunikation“ } \\
\text { (HYMES 1979), „wonach die Ausdrucksvariation zwischen } \\
\text { verschiedenen Individuen oder Gruppen im Sinne kulturel- } \\
\text { ler Unterschiede betrachtet wird“ (KEIM 1997:319). } \\
\text { Im Rahmen dieses Projekts wird die kommunikative Praxis } \\
\text { türkischstämmiger Jugendgruppen aus Ausländerstadttei- } \\
\text { len Mannheims erforscht. Den Kern der Untersuchung stel- } \\
\text { len deutsch-türkische Sprachmischungen, die den Sprach- } \\
\text { und Kommunikationsstil der drei Migrantengruppen, und } \\
\text { zwar den der „Europatürken“ (AsLAN 2005), der „türki- } \\
\text { schen Powergirls“ (KEIM 2008) und der „Unmündigen“ } \\
\text { (eine Gruppe „emanzipatorischer Migranten“ [Eigenbe- } \\
\text { zeichnung] mit einem speziellen Migrantendeutsch: CiN- } \\
\text { DARK 2005, 2010) kennzeichnen. }\end{array}$ \\
\hline 3. & Das Stuttgarter Projekt & $\begin{array}{l}\text { Dieses Projekt läuft unter dem Titel „Jugendsprache in } \\
\text { Stuttgart unter dem Einfluss von kultureller, ethnischer und } \\
\text { sprachlicher Vielfalt“ und erforscht eine „,ethnolektale Va- } \\
\text { rietät der Standardsprache, die von einer ethnischen Min- } \\
\text { derheit typischerweise verwendet wird“ (AUER 2005). } \\
\text { Ausgangspunkt dieser Untersuchung war die Hypothese, } \\
\text { dass soziale Netzwerke das Sprachverhalten Jugendlicher, } \\
\text { die sprachbewirkten ,acts of identity“ (DIRIM / AUER 2004) } \\
\text { prägen. Falls die besagten Netzwerke multiethnische } \\
\text { Strukturen besitzen, können darin verwendete Ethnolekte } \\
\text { auf den allgemeinen Jugendsprachstil einwirken und im } \\
\text { Endeffekt die Etablierung/Herausbildung einer neuen } \\
\text { Sprachvarietät herbeiführen. Sofern der ethnische Sprech- } \\
\text { stil also nicht lediglich auf ethnische Gruppen beschränkt } \\
\text { und eher zu einem allgemeinen „Usus“ in multiethnischen } \\
\text { Lebenswelten geworden ist, ist von einer „De-Ethnisie- } \\
\text { rung“ des Ethnolekts die Rede (vgl. AuER 2003, DIRIM / } \\
\text { AuER 2004). Darüber hinaus spricht die Übertragung der }\end{array}$ \\
\hline
\end{tabular}




\begin{tabular}{|l|l|}
\hline & $\begin{array}{l}\text { deutsch-türkischen Sprachvariante auf deutschstämmige } \\
\text { Jugendliche Auers Erachtens nach deutlich dafür, dass es } \\
\text { sich mittlerweile um einen Soziolekt des Deutschen han- } \\
\text { delt. }\end{array}$ \\
\hline
\end{tabular}

Die Nachlese der sprachwissenschaftlichen Leistungen sind Medienbeiträge schlechthin. Sie werden von Mitwirkenden der Projekte sowie sprachorientierten Journalisten, und zwar teils mit einer besonderen Teilnahme von Experten konstruiert und einer breiten internationalen Leserschaft zugänglich gemacht. Bemerkenswert ist im engen Zusammenhang damit, dass Massenmedien nicht nur als Schauplatz zur Auseinandersetzung mit normwidrigen Sprachpraktiken, sondern auch (und vielleicht insbesondere) als eine Art Motor für deren Etablierung und Weiterentwicklung in der nicht ausschließlich bundesdeutschen Gesellschaft fungieren. Die mediengesteuerte Reaktion auf den ursprünglichen (,primären“) Ethnolekt mündet in die Produktion von Konstrukten mit einem grundsätzlich unterhaltsamen Charakter, worin die salientesten ethnolektalen Sprachmerkmale (wie bspw. Koronalisierung palataler Frikative, Ausfall von Artikeln und Präpositionen in Lokal- und Richtungsangaben) aufgegriffen, frei erweitert, hochgradig stilisiert und im komischen Modus verarbeitet werden. Gewöhnlich sind es, wie bereits zuvor nahegelegt, comedy acts (vgl. DIRIM / AUER 2004), die ethnolektale Sprachstrukturen vereinnahmen und in übersteigerter Form veranschaulichen:

TV-Komiker wie Erkan und Stefan oder Kaya Yanar (,Was guckst du“) etablierten das Türkendeutsch als leicht debiles Gestammel schrulliger Machos: Längst ist das latent diskriminierende Mimen goldkettchentragender Türkenprolls zum gern verwendeten Stilmittel im Alltagsdeutsch geworden. In Comedy-Shows wird die sogenannte „Kanak-Sprak“ als Ethno-Gag vermarktet, werden die immer gleichen „vollkrassen“ Sprüche à la Erkan und Stefan zitiert - im Glauben, dass Jugendliche in Neuperlach genau so reden. Doch Sprüche wie „Ich mach dich Messer“ oder „Ich mach dich Krankenhaus“ sind nichts anderes, als ein stilisiertes, kabarettistisches Kunstprodukt. (LÜBER 2006:1)

Der medial popularisierte (,sekundäre') Ethnolekt wird von der bundesdeutschen Jugend übernommen (in das eigene Sprachregister eingebettet) und mit einer auffälligen Betonung (einem scherzhaft-spöttischen Unterton) zur Abgrenzung von Sprechern des ,primären' Ethnolekts verwendet. Somit ist der ,tertiäre‘ Ethnolekt das Ergebnis der medialen Verbreitung und Popularisierung des ,Ghettodeutschen“ schlechthin. Vor dem besagten Hintergrund gelten die ,mediale‘ und ,tertiäre‘ Ausprägung des ethnolektalen Sprechstils als eine Art ,Transgression` (sprachlicher ,Grenzüberschreitung'), die die ,Übernahme‘ einer als ,fremd‘ empfundenen Stimme bedeutet (vgl. DiRIM / AuER 
2004:39). Mit dem ,primären` Ethnolekt an sozialen Brennpunkten hat dies aber nicht viel zu tun:

Völlig konträr zum Klischeebild des stammelnden Türstehers oder einsilbig aggressiven Machos kultivieren gerade Einwandererkinder aus der Mittelschicht ein besonders eloquentes Deutsch. So kann es passieren, daß der Sohn des türkischen Eckladenbesitzers den verdutzten Kunden mit Höflichkeitsfloskeln überrascht, die mittlerweile selbst bei Muttersprachlern Seltenheitswert haben: „Ich habe zu danken, der Herr!“ (LÜBER 2006:1)

Alles in allem ist der ursprüngliche Ethnolekt „so vielfältig wie die sozialen Schichten, in denen er gesprochen wird“ (LÜBER 2006:1). Eine hohe Variabilität von ethnolektalen Sprachmerkmalen bei ein und demselben Sprecher, gepaart mit einem fließenden Hin- und Herwechseln zwischen dem städtischen Substandard und dem Standarddeutschen (als eine weitere Stilmöglichkeit, um sich auszudrücken), lassen die besagte Mischvariante nur bedingt als Bedrohung bzw. eine dauerhafte Verarmung des gesprochenen Deutsch wahrnehmen. Eine These, die allerdings nicht von allen Linguisten und Sprachjournalisten goutiert und demnach zum Gegenstand der medial geführten Polemik wird.

\section{Ergebnisse der Fallstudien und mediengesteuerte Reaktionen}

\subsection{Die mediale Berichterstattung zu HEIKE WIESES Kiezdeutsch}

Genau zwei Jahre vor der Veröffentlichung von HEIKE WIESES Kiezdeutsch ein neuer Dialekt entsteht (2012) verfasst die Autorin und Potsdamer Linguistin in einem sechs Seiten langen Beitrag ihre Grundthesen zur ,Kiezdeutsch“. Der skizzenhafte Überblick über diese Sprachvariante beleuchtet im Wesentlichen, was sich hinter dem Terminus ,Kiezdeutsch“ eigentlich verbirgt. Demnach referiert der Begriff auf einen Kommunikationsstil Jugendlicher deutschund fremdstämmiger Herkunft, die in sozialen Brennpunkten deutscher Großstädte (Berlinisch: ,Kiez`) wohnen und miteinander agieren. WIESE sieht den Interaktionsstil in ethnisch gemischten Gruppen als einen deutschen (im System des im Standarddeutschen verankerten) und im Übrigen in sich stimmigen Dialekt (Multiethnolekt nach CLYNE 2000) an. Demnach sei ,Kiezdeutsch“ weder exotisch noch ein Ausländerdeutsch. Doch der Multiethnolekt aus dem ,Kiez‘ wird im überwiegenden Maße von Jugendlichen, die in Deutschland geboren und aufgewachsen sind, gesprochen und von ihnen meist als eine einem breiteren Varietätenspektrum entnommene Variante des Deutschen 
empfunden und vor allem bei der Interaktion mit Gleichgesinnten eingesetzt. Im Hinblick darauf führt die Linguistin Folgendes aus:

Man muss sich hier [aber] klar machen, dass es nicht das eine Deutsch gibt, sondern dass die deutsche Sprache, wie jede Sprache, ein Spektrum unterschiedlicher Dialekte und Stile umfasst, und die Entwicklung von Jugendsprachen ist ein Aspekt davon. Wir alle beherrschen mehrere Elemente dieses Spektrums und sprechen beispielsweise neben dem Standarddeutschen noch eine regional gefärbte Varietät oder einen Dialekt, und wir sprechen ein stärker umgangssprachliches Deutsch mit der Familie oder mit Freunden als etwa mit Vorgesetzten oder bei einer Prüfung. Ebenso ist Kiezdeutsch nur ein Teil des sprachlichen Repertoires von Jugendlichen: Kiezdeutsch wird unter Freunden gesprochen, aber nicht mit Eltern, Lehrern usw. Kiezdeutsch stellt somit grundsätzlich keine Bedrohung für das Deutsche dar: Auf der Ebene seiner Sprecher ist es Teil eines größeren sprachlichen Repertoires, auf der Ebene des Sprachsystems einer von vielen Dialekten des Deutschen - lediglich mit der Besonderheit, dass es sich hier um einen Dialekt handelt, der überregional im multiethnischen urbanen Raum beheimatet ist. (WIESE 2010:2)

Des Weiteren sei ,Kiezdeutsch` kein isoliertes Sprachphänomen. Denn gleiche sprachliche Gegebenheiten wurden bereits in anderen europäischen urbanen und multiethnischen Sprachräumen (vgl. die ,Straattaal' [Straßensprache] in Amsterdam, das ,Rinkebysvenska“ [Rinkeby-Schwedisch] in Stockholm oder der ,Københavnsk Multiethnolekt‘ [Kopenhagener Multiethnolekt] in Dänemark) festgestellt und ausführlich belegt (vgl. hierzu WIESE 2012:109-126). WIESES Erachtens sind multiethnolektale Eigenschaften (wie etwa Wegfall von Artikeln und Präpositionen in Orts- und Richtungsangaben; Zusammenziehungen, die mitunter die Form einer Partikel annehmen: ,lassma', ,musstu', ,ischwör‘; der übermäßige Gebrauch von ,so“ als Intensivierer und Fokusmarker sowie von desemantisierten verstärkenden Adjektiven und Adverbien wie ,krass', ,voll‘, ,geil‘) bereits existierende Tendenzen des Deutschen, die mit dem ,Kiezdeutsch' nur verstärkt ins Blickfeld geraten. Dies ist aus der folgenden Anmerkung WIESEs abzuleiten:

Grundsätzlich können wir in der Entwicklung des Deutschen (und nicht nur dort) die Tendenz beobachten, dass Flexionsendungen und funktionale Elemente wie der Artikel „ein“ verkürzt werden oder entfallen. So heißt es im heutigen Deutsch nicht mehr „dem Manne“, sondern „dem Mann“, ohne nominale Kasusendung; im gesprochenen Deutsch entfallen Personalendungen von Verben häufig in der ersten Person Singular, etwa „ich sag“ statt „ich sage“, und der indefinite Artikel „ein“ wird oft stark reduziert und an das vorhergehende Wort gehängt: „Hast du 'n Handy?" Diese Tendenz des Deutschen spiegelt sich auch in Kiezdeutsch wider: Die Verkürzungen, die wir dort finden, sind im System des Deutschen bereits angelegt; sie bringen kein fremdes Element der grammatischen Reduktion von 
außen hinein, sondern führen eine bereits vorhandene sprachliche Entwicklung des Deutschen weiter. Eine solche Weiterführung ist charakteristisch für Dialekte, die oft dynamischer und innovativer sind als die Standardsprache, da sie keiner so starken schriftsprachlichen Normierung unterworfen sind. (WIESE 2010:2f.)

Im Grunde stehe ,Kiezdeutsch“ für einen kreativen Umgang mit Sprachelementen und eine sprachliche Innovation und Bereicherung, was aufgrund der Integration von Neuzugängen aus den Herkunftssprachen Andersstämmiger in das deutsche Sprachsystem vollzogen wird:

Erstens werden neue Wörter verwendet, die etwa aus dem Türkischen oder Arabischen stammen wie „Lan“ (wörtlich „Mann/Typ“) oder „wallah“ (wörtlich „und Allah"). Diese Ausdrücke machen Kiezdeutsch nicht zu einer türkisch-deutschen oder deutsch-arabischen Mischsprache, wie manchmal angenommen wird, sondern werden als neue Fremdwörter integriert: Sie werden nach den Regeln der deutschen Grammatik verwendet („Lan“ zum Beispiel so ähnlich wie „Alter“ in der Jugendsprache, „wallah“ so ähnlich wie „echt“), und ihre Aussprache wird eingedeutscht. (WIESE 2010:2)

Vor dem besagten Hintergrund mag ,Kiezdeutsch' kein falsches, schlechtes Deutsch sein, das auf Unsicherheit und Unwissenheit seiner Sprecher beruht. Es wird von der Linguistin als ,akzeptabel‘ und ,legitim‘ empfunden und so dargestellt, wenn sich dieser ethnisch geprägten Mischvariante bedienende Interaktanten in den Regeln des Standarddeutschen auskennen und diese auch umsetzen können. Sofern ,Kiezsprecher' also in ihrem Sprachrepertoire präsente Abweichungen vom Standarddeutschen erkennen und in schulischen oder beruflichen Kontexten korrigieren oder aber auch ablehnen können, gelte ,Kiezdeutsch“ als ,harmlose“ und ,nicht [automatisch] stigmatisierende“ Sprechart ethnisch gemischter Gruppen. Mit einem derartigen Deutungsmuster stellt sich die Linguistin aber vorwiegend auf erboste Reaktionen ein. Diese werden durch systematisch in Medien auftretende kiezdeutschtypische Floskeln und Drohrituale des Typs ,Hast du U-Bahn?‘, ,Lassma treffen“, ,Machst du rote Ampel', ,Was guckst du, bin isch Kino?', ,Isch mach disch Messer! ‘ verschärft und intensiviert. Letztendlich werden der ,Kiezdeutsch'-Erfinderin rassistische Tendenzen unterstellt, weil sie mit ihrem linguistisch abgesicherten Plädoyer für das Standardferne sozial benachteiligten ,Kiez'-Bewohnern die Fähigkeit zur Aneignung von normkonformen Sprachstrukturen abspreche. Dies bringt der Journalist MatTHIAs Heine wie folgt auf den Punkt:

Offenbar dämmert es Heike Wiese keine Sekunde, dass der wahre Rassismus darin bestehen könnte, Jugendlichen mit türkischem oder arabischem Migrationshintergrund die Fähigkeit abzusprechen, korrektes Standarddeutsch zu lernen. Aber wenn die Professorin Sätze wie „Machst du rote Ampel?“ für „innovativ“ erklärt und nahelegt, solche Konstruktionen, die auf Präpositionen und Artikel 
verzichten, stünden gleichwertig neben schulgrammatisch korrekten Formulierungen, dann verfolgt sie damit eine klassische linke Onkel-Tom-Strategie. Im Bildungsbereich besteht in diesem politischen Lager nämlich immer die Tendenz, alles, was für ihre marginalisierte Klientel zu schwierig sein könnte, als überflüssiges Herrschaftswissen zu diffamieren, dessen geheimer Zweck darin besteht, die Klassenunterschiede auch sprachlich zu zementieren. Im Grunde ihres Herzens hält Frau Wiese offenbar Migranten für zu dumm und zu verweichlicht, um die Härte eines traditionellen Deutschunterrichts aushalten zu können. Sie erniedrigt die so Geschützten aber allesamt zu sprachlichen „Onkel Toms“, also zu lustigen Zurückgebliebenen, auf die die Mehrheitsgesellschaft wohlwollend herabsieht. [...] Je höher die Bildung, desto eher besteht der paradoxe Trick darin, Kiezdeutsch zu sprechen und sich gleichzeitig von dessen originären Sprechern zu distanzieren. Im Grunde ist das wie „Blackfacing“. So nennt man in Amerika die lange von Weißen verübte rassistische Humorpraxis, sich das Gesicht schwarz anzumalen, um über die Schwarzen und ihre kulturellen Besonderheiten zu spotten. Wenn Deutsche, die es besser können, Kiezdeutsch reden, ist das oft „Turkfacing“ - man schminkt sich sprachlich als Türke, um unter den biodeutschen Freunden ein paar leichte Humorpunkte zu machen. (HeINE 2014:1)

\subsection{Die mediale Berichterstattung zu UWE HINRICHS’ Multi Kulti Deutsch}

Der Leipziger Sprachwissenschaftler UwE HINRICHS unterschlägt keineswegs, dass der eigentliche Anreiz für sein Buch Multi Kulti Deutsch. Wie Migration die deutsche Sprache verändert (2013) sein im Spiegel-Magazin publizierter Essay (samt der regen Reaktionen, die dieser auslöste) war. Der Essay (HINRICHS 2012) heißt: Hab isch gesehen mein Kumpel und ist mit dem Untertitel Wie die Migration die deutsche Sprache verändert hat versehen. Hierzu äußert sich HINRICHS in Vorbemerkungen zu seinem Buch wie folgt:

Dieses Buch ist entstanden aus einem Essay, den Der Spiegel im Februar 2012 veröffentlichte, und hier vor allem aus den gegensätzlichen und zum Teil überschäumenden Reaktionen, die er hervorrief. [...] Die Reaktionen auf jenen Essay fielen offenbar vor allem deswegen so heftig, so ambivalent und so schroff aus, weil dieses Thema über Jahrzehnte ein subtiles, wohlgehütetes Tabu war und noch immer ist - jedenfalls in der öffentlichen Kommunikation und in der linguistischen Wissenschaft. Ein essentielles, nagendes, latentes Problem der deutschen Gesellschaft drängte aus dem Dunkel des Unbewussten an die helle Oberfläche der Diskurse und der Medien und wollte gehört werden. Denn immerhin gaben 84 Prozent der Deutschen im Jahre 2010 an, sie bemerkten deutliche Veränderungen ihrer Sprache, und fast ein Drittel führte dies diffus auf den Einfluss auf Migrantensprachen zurück. Eben weil hier weithin die Unklarheit herrscht, gab es aus dem Volk höchst ambivalente Reaktionen: seltsame und kuriose Fehldeutungen, absurde oder abstruse Missinterpretationen, ganz banale Missverständnisse, viele Unterstellungen und hier nichtwiederzugebende Kommentare und Anschuldigun- 
gen. Auf der anderen Seite gab es auch ordentlich Beifall und eine gewisse breitstreuende Genugtuung darüber, dass ein Tabuthema von einer ungewohnten, ganz neuen Seite beleuchtet wurde. Auch Lob von der falschen Seite war natürlich dabei, und barsche Kritik von jenen, die sich selber für sprachlich sensible Zeitgenossen hielten. (HINRICHS 2013:9f.)

In den Vordergrund des besagten Essays rückt ein Sprachwandel, der seit den siebziger Jahren im bundesdeutschen Kontext an Fahrt gewinnt. Der ,rasant beschleunigte Sprachwandel' sei in überwiegendem Maße ,kontaktinduziert': „Die Veränderungen sind kontaktinduziert und gehen deshalb um ein Vielfaches schneller vor sich, als es ohne Fremdspracheneinfluss der Fall wäre. Das Erste, was eine Sprache verliert, ist das, was sie für einfache Kommunikationszwecke mit fremden Sprechern am allerwenigsten benötigt: Das sind die Fälle, die Endungen und die Regeln ihrer Verknüpfung. Was man nicht braucht, das schleift sich schnell ab“ (HINRICHS 2012:104). HiNRICHS’ Erachtens beziehen sich die Veränderungen grundsätzlich auf das gesprochene Deutsch: „Es verändert sich vor allem das gesprochene Deutsch, also die Umgangssprache, weil die alltägliche Mehrsprachigkeit fast ausschließlich mündlich auftritt“ (HINRICHS 2012:104). So betrachtet sind zahlreiche und gewöhnlich in der Morphosyntax abgezeichnete Normabweichungen eine Art Kompromiss, den Migrierte und Nichtmigrierte eingehen, um möglichst effizient miteinander kommunizieren zu können. Dies geht zumindest aus dem folgenden Essay-Ausschnitt hervor: „Das mehrsprachige Milieu kann auf korrekte Deklination und genaue Endungen durchaus verzichten, weil diese Art Grammatik nur Kodierungsenergie frisst, die woanders viel dringender gebraucht wird, beispielsweise um Defizite im Wortschatz auszugleichen“ (HINRICHS 2012:104f.). Nun aber liegen hinter einem derartigen ,kontaktinduzierten“ Sprachwandel Vermischung und Vermengung von Sprachstrukturen verborgen. Muttersprachler greifen auf Satzbausteine und Satzmuster zurück, die einem anderen Sprachtyp angehören und eignen sie sich an (,foreigner talk'). Im Endeffekt sind wir Zeugen einer Phase, „in der ,falsches“ und ,richtiges“ Deutsch in Konkurrenz liegen und beide Varianten oft direkt nebeneinander geäußert werden - ohne dass die Sprecher sich dessen bewusst wären“ (HINRICHS 2012:105). Als prägnantes Beispiel hierfür wird von HINRICHS der deutsche Ethnolekt genannt:

Im großstädtischen Kiezdeutsch, das die Potsdamer Linguistin Heike Wiese erforscht, gibt es etwa eine Vielzahl von Satzmustern, die aus dem Arabischen oder Türkischen stammen, etwa die Wortfolge („Hab isch gesehen mein Kumpel gestern!“) oder das Fehlen der Präposition („Ich geh Schule“). Auch in der AlltagsUmgangssprache kündigen sich bereits deutlich Tendenzen an, die von vielen 
Migrantensprachen gestützt werden: eine neue Steigerung mit „mehr" („mehr geeignet“, „mehr zuständig“) oder der Zusammenfall von Ort und Richtung („die Politiker fuhren letzte Woche auf einem Finanzgipfel“) sind Beispiele für neue Strukturen, die ihre Vorbilder in vielen Migrantensprachen haben. Auch die zahlreichen neudeutschen Ausdrücke mit „machen“ wie „einen Film machen“, „ein Tor machen“, „einen Kompromiss machen“ haben direkte Parallelen im Türkischen. (HINRICHS 2012:105)

Da sich die Grenzen zwischen normkonformen und normabweichenden Sprachmustern stets verschieben und verschwimmen, lässt bei Muttersprachlern die Bereitschaft nach, diesem Trend entgegenzuwirken, d.h., die Fehler auch als Fehler anzusehen und nötige Korrekturen einzubringen. Auf Dauer mündet dieser Prozess in Etablierung und Stabilisierung von neuen Sprachstrukturen, denen Sprachkreuzungs- und Mischungsprozesse im Migrantenmilieu ihren Stempel aufgedrückt haben. Diese ,Drift‘ gilt als Gegenstand der neuerdings aufgekommenen und von HINRICHS erforschten Disziplin ,Eurolinguistik'.

Die Nachlese von HINRICHS Erwägungen über migrationsbedingte dauerhafte Veränderungen des gesprochenen Deutsch sind Feuilletons, in denen die von dem Linguisten vorgeschlagene Argumentation mitunter nur bedingt goutiert wird. Hierbei wird jeweils zunächst das von HINRICHS prognostizierte Zukunftsdeutsch entschlüsselt:

„Die Sprache von meine Vorfahr war mehr kompliziert wie heut.“ So etwa klingt das Hochdeutsch der Zukunft. Das besagt die Prognose von Uwe Hinrichs, Linguistikprofessor an der Universität Leipzig. (KRISCHKE 2006:1)

Es gibt Turbowandel von deutsche Sprache und kann jeder beobachten schon in sein Alltag ein starker Trend auf mehr einfach. Das in etwa ist die Form, die nach Uwe Hinrichs' Meinung das Deutsch einer gar nicht mehr so fernen Zukunft haben wird. Die Kasusendungen sind abgeschliffen, grammatische Übereinstimmungen zwischen den Wörtern im Satz spielen kaum noch eine Rolle, Präpositionen stehen zur beliebigen Verwendung, das grammatische Geschlecht ist eingedampft, der Konjunktiv geht den Bach hinunter, die Satzstrukturen versimpeln kurz: vom Formenreichtum und von der Regelstrenge des heutigen Deutsch wird, jedenfalls in seiner mündlichen Existenzform, kaum noch etwas übrig sein. (KRISCHKE 2013:1)

Nun aber wird die Mehrsprachigkeit als Motor für sprachliche Normverstöße sowie deren Häufigkeit aufgrund mangelnder empirischer Belege HINRICHS' angefochten: „Empirische Belege für die tatsächliche Häufigkeit dieser Normabweichungen bleibt Hinrichs hier wie auch sonst schuldig. Seine spekulative Behauptung, große Korpora gesprochener Sprache würden - wenn es sie denn 
gäbe - seine Aussagen ,ohne Zweifel erweisen', darf man durchaus bezweifeln“ (KRISCHKE 2013:2). Auch die meisten Sprachveränderungen, die HINRICHS in seinen Publikationen angeht, haben, wie bereits zuvor nahegelegt, noch lange vor der derzeitigen Migrationswelle in der Bundesrepublik Deutschland bestanden:

Die Vereinfachung der Flexion mit abbröckelnden grammatischen Endungen ist seit indogermanischen Zeiten im Gange, über die Verwechslung der Präpositionen, die Verhunzung des Konjunktivs und die Verdrängung des Genitivs durch Konstruktionen mit ,von“ wetterte schon Arthur Schopenhauer, und Wendungen wie „der Mutter ihr Hut“ gehören zum dialektalen und umgangssprachlichen Altbestand des Deutschen. Zwar erwähnt auch Hinrichs, dass es einen solchen langfristigen Sprachwandel gibt, ohne aber zu klären, welche Rolle vor diesem Hintergrund der Einfluss der Migrantensprachen nun eigentlich genau spielt. (KRISCHKE 2013:3)

Als neu und bemerkenswert wird von dem Journalisten WOLFGANG KRISCHKE nicht die Existenz normabweichender und sich jeweils aus Herkunftssprachen Hinzugezogener speisender Strukturen, sondern vielmehr ihre ,mediale Dauerpräsenz‘ empfunden. Die medial ,abgesicherte‘ Akzeptanz und Hervorhebung von sprachlichen Normverstößen sei stärker als der Einfluss der Migrantensprachen selbst. Die mögen seines Erachtens zwar „ein Rädchen im Getriebe des Sprachwandels, aber nicht ihr Motor“ (KRISCHKE 2013:3) sein.

\subsection{Die mediale Berichterstattung zu DiANA MAROSSEKs Kurzdeutsch}

In Anlehnung an HiNRICHS sieht die Berliner Soziolinguistin DiANA MAROSSEK dauerhafte Veränderungen im gesprochenen Deutsch (und zwar innerhalb von 15 Jahren) voraus. Um zu diesem Schluss zu kommen, hat MAROSSEK eine intensive Feldforschung in Berlin betrieben: Getarnt als Referendarin besuchte sie „dreißig über ganz Berlin verteilte Schulen aller Typen und notierte dort die Unterrichts- und Pausengespräche von Acht- und Zehntklässlern. Von dieser Expedition durch die jugendsprachliche Landschaft von der Hauptschule in Wedding bis zum Gymnasium in Steglitz hat sie eine beeindruckende Menge sprachlicher Daten ${ }^{1}$ mitgebracht“ (KRISCHKE 2016:2). MAROSSEKS

„Mein Ziel war es, in jedem Berliner Teilbezirk mindestens eine Schule zu untersuchen. Am Ende standen auf meiner Liste 30 Schulen in ganz Berlin, die ich jeweils einen ganzen Schultag lang in den 8 . und 10. Klassen besuchte. Insgesamt sollte ich auf diese Weise 1395 Schülern in 78 Unterrichtsstunden und wahrscheinlich noch mal doppelt so vielen auf den Pausenhöfen beim Sprechen zuhören. Akribisch dokumentiert und ausgewertet, hat das am Ende eine solide Doktorarbeit von 400 Seiten ergeben - eine der größten Sprachstudien in Deutschland 
Doktorarbeit mit dem Titel Gehst du Bahnhof oder bist du mit Auto? Warum wir reden, wie wir neuerdings reden brachte der Verfasserin den 2. Platz des Deutschen Studienpreises 2014 der Körber Stiftung und enorme Medienbeliebtheit ein.

Im Gegensatz zu der Behauptung HinRicHs' sind Reduktionen und Simplifizierungen in der deutschen Morphosyntax nach MAROSSEK nicht nur (und nicht in erster Linie) auf Migrationsprozesse und die damit einhergehende Mehrsprachigkeit zurückführbar. Diese werden nämlich, so wie bei WIESE, durch lokale und dialektale Einflüsse, aber auch einen medialen Input (Ethnolekt als Szenesprache) mitgeprägt:

Dabei hat Kiezdeutsch weder in Berlin noch in anderen deutschen Städten automatisch etwas mit Migration zu tun. [...] Ob im tiefbürgerlichen Zehlendorf oder in den Migrantenvierteln Neuköllns - [sie; Marossek] fand keine großen Unterschiede. Überall fielen Sätze wie „Kommst du mit Klo?“ oder „Ich war Fußball“. [...] Nur von türkischen Klassenkameraden hätten diese Teenager ihr Kiezdeutsch dabei nicht abgekupfert, ist Marossek überzeugt. Denn auch die „Berliner Schnauze“ liebe das Verkürzen und Weglassen von Artikeln und Präpositionen. „Auf Schicht sein“ kennt aber auch das Ruhrgebiets-Deutsch. Dort sind Grammatikkonstruktionen wie „Tu ma die Mama winken“ oder „Meine Oma ihre Tasche“ nicht nur ein Fall fürs Kabarett. Für Marossek haben sich damit zwei ähnliche Trends - deutsche Dialektgrammatik und Übernahmen aus der Muttersprache von Migranten - gefunden und verbunden. (VON LESZCZYNSKI 2014:1)

Im Vordergrund ihres linguistischen Interesses stehen also Kurzformen ( $\mathrm{Zu}-$ sammenziehungen aus Präpositionen und Artikeln wie bspw. am, beim, ins, vom, zur, zum), die zwar bei anderen Linguisten als durchgängiges Merkmal ethnolektalen Sprechstils aufgezeigt und beschrieben wurden, hier aber die neue Bezeichnung „Kontraktionsvermeidungen“ (MAROSSEK 2016:16) erfahren. Im Zusammenhang damit merkt KRISCHKE Folgendes an: „Einige Beispiele, die sie anführt (,Wir fahren Genf‘, ,Er ist auf Arbeit‘), zeigen allerdings einen laxen Umgang mit dem Begriff ,Kontraktionsvermeidung', denn hier werden zwar Präpositionen oder Artikel, aber keine Kontraktionen ausgespart“ (KRISCHKE 2016:1). In Bezug auf die besagten Schrumpfformen nennt MAROSSEK das in Berlin erforschte Sprachphänomen nicht mehr ,Kiezdeutsch', sondern „Kurzdeutsch“ (MAROSSEK 2016:16). Die ehemalige Bezeichnung habe sich ihres Erachtens in fachlinguistischen Kreisen kaum durchgesetzt, löse negative Konnotationen aus und deute schließlich auf sprachliche Veränderungen lediglich innerhalb und nicht außerhalb des Migrantenumfeldes hin

und die bislang einzige zum aktuellen Wandel der deutschen Umgangssprache.“ (MAROSSEK 2016:20). 
(vgl. Heine 2016:1, MAROSSEK 2016:12-14). Hierbei wird der ethnolektale Sprechstil längst von aufstiegsorientierten und darüber hinaus nicht unbedingt jugendlichen Sprechern ohne Einwanderungsbiographie verwendet. Denn laut MAROSSEKS Angaben wird das mittlerweile als identitätsstiftendes Symbol fungierende ,Kurzdeutsch“ sogar an Gymnasien zum festen Bestandteil der sprachlichen Gruppenpraxis, das von Schulpädagogen nicht korrigiert, sondern gerade umgekehrt sogar in ihren eigenen Sprachgebrauch eingebettet wird:

Doch selbst Lehrer, die sich zuerst über Kiezdeutsch amüsierten, hätten später unwillkürlich Artikel weggelassen. Dazu gibt es Kostproben in der Doktorarbeit. Schüler Sebastian sagt: „Ich brauche Locher!“ Und seine Lehrerin antwortet: „Ist Locher nicht vorne drin?“ (VON LESZCZYNSKI 2014:1)

Und selbst im Lehrerzimmer schnappte die Sprachforscherin einen solchen Satz auf: „Karin, ich geh mal schnell Bäcker, willst Du was?“ (HARTwIG 2014:1)

Offenbar darf MAROSSEKS Überzeugung von einer derartigen Schulroutine, die obendrein auf einzelnen Beobachtungen, subjektiven Eindrücken und Annahmen und nicht systematischen Untersuchungen beruht, nicht ernst genommen und folglich auch abgelehnt werden. Die zuhauf dokumentierten Belege für normabweichende Sprachpraktiken Jugendlicher mit und ohne Migrationsgeschichte, und zwar in schulischen und außerschulischen Kontexten, sind jedoch schwer anfechtbar. In Bezug auf die ,Kontraktionsvermeidungen“ ist jedoch auffällig, dass die Soziolinguistin ,verschmolzene‘ und ,nichtverschmolzene' Formen als gleichrangig betrachtet, ohne richtig einzusehen, dass ,zum Bahnhof‘ zu kommen etwas anderes als ,zu dem Bahnhof‘ zu kommen bedeutet (vgl. KRISCHKE 2016:1). Dabei wäre die Übertragung solcher Nuancen ins ,Kurzdeutsch“ hochinteressant, gibt der Journalist zu bedenken (vgl. KRISCHKE 2016:1). Glücklicherweise beabsichtigt MAROSSEK eine weitere Recherche zum Sprachwandel in anderen Regionen Deutschlands: „Ich habe gehört, dass in Köln ähnliche Beobachtungen über die Jugendsprache gemacht werden. Das will ich genauer wissen.“ (HARTWIG 2014:2) und würde damit ihr grundsätzlich auf den Ergebnissen ihrer Doktorarbeit basierendes Befundspektrum erweitern, was zur Schließung der vorstehend angesprochenen Lücke beitragen könnte.

\subsection{Gastbeiträge und Gastkommentare}

Das Aufrollen von brisanten Themen wie dem rasant beschleunigten Sprachwandel erfolgt nicht nur über Beiträge eigener oder fremder Autorschaft. Für eine derartige Auseinandersetzung scheinen Interviews mit einer besonderen Teilnahme von den Initiatoren (Provocateurs) der Dauerbrenner-Themen mitunter sogar viel geeigneter zu sein. Unverblümt, in einem Plauderton trotz der 
Schwere der zu vermittelnden Botschaft können relevante und ansonsten aus Gründen der political correctness nicht aufgegriffene Sachverhalte besser und nachhaltiger vermittelt und infolgedessen auch nachvollzogen werden. Untervier-Augen-Gespräche ermöglichen es nämlich, sich zunächst gewisser grundlegender Sachverhalte zu vergewissern, um sie dann vor Ort aufgeklärt zu bekommen. Offenbar kann man sich als Interviewte(r) vor Fragen gestellt sehen, die man sich selbst zuvor nicht gestellt hat oder die die Grenzen der Publikation sprengen würden. Diese erfordern in Interviews eindeutige und nicht ausweichende Antworten sowie blitzschnelle Reaktionen, selbst wenn sie für den Interviewten die Überschreitung seiner Privatsphäre bedeuten. So lernt man aber auch die Persönlichkeit des Betroffenen besser kennen, was gewissermaßen die Identifizierung oder Nichtidentifizierung mit seinen Wahrnehmungsund Deutungsmustern impliziert. Kurzum: Im entspannten Rahmen der Interviews kristallisieren sich eigene und fremde Standpunkte, die eingangs schon genannten Thesen können erfolgreich verteidigt, Einwände gekontert und verbale Angriffe pariert werden. All dies findet in zwei unten veranschaulichten Interview-Ausschnitten statt, die unter dem ethnolektalen Sprechstil Jugendlicher (aber auch Erwachsener) in Deutschland einen gemeinsamen Nenner finden:

Tabelle 3

\begin{abstract}
Jugenddialekt Kiezdeutsch: „Ich bin Alexanderplatz“"

Unvollständige Sätze, durchmischt mit arabischen und türkischen Worten: Kiezdeutsch, die Jugendsprache der Großstädte, hat keinen guten Ruf. Zu Unrecht, sagt Sprachwissenschaftlerin Heike Wiese: Der Slang wird als Dialekt des Deutschen erhalten bleiben.

Süddeutsche.de, 8.02.2012, Interviewer: MARC FELIX SERRAO
\end{abstract}

SZ: „Hallo, ich bin die Heike, und ich habe gerade ein Buch geschrieben“ - können Sie das bitte mal auf Kiezdeutsch sagen, Frau Wiese?

HEIKE WIESE: Der Bitte kann ich kaum entsprechen. Kiezdeutsch ist ja Deutsch. Das ist nicht exotisch.

SZ: Bin isch Heike, hab isch Buch geschrieben, Moruk.

WIESE: Ja, das . . . ginge. Es wäre der Situation aber überhaupt nicht angemessen. Unserem Alter auch nicht. Das würde ich vielleicht
Herr Lehrer geht Kopierer

Eine Soziolinguistin erforscht, wie sich Berliner Schüler Türkisch aneignen.

Die Zeit Nr. 27/2014, 10.07.2014, Interviewführer: BURKHARD STRASSMANN

DIE ZEIT: Ihre Doktorarbeit trägt den Titel „Gehst du Bahnhof oder bist du mit Auto?“ Wo sind Sie diesem Satz begegnet?

Diana MARosseK: Zu Hause! Meine jüngere Schwester hatte Freundinnen zu Besuch, alle von einer behüteten Schule in Pankow am Stadtrand von Berlin, ohne Migrationseinflüsse. Trotzdem sprachen sie, als ob sie aus dem tiefsten Wedding kämen. Ich habe auch Kita-Erzieherinnen mit ihrer Gruppe so sprechen gehört oder Menschen in der S-Bahn. 
sagen, wenn ich ein Teenie wäre. Aber auch dann reden die ja so nicht mit Erwachsenen.

SZ: Wie alt sind Kiezdeutschsprecher?

WIESE: Das sind Jugendliche, also etwa zwölf bis 20. Ein Wort wie „Moruk“ (Türkisch für „Alter“, die Red.) würde aber auch von denen nicht jeder verwenden. Die Jüngeren kennen es noch nicht, und die Älteren benutzen es, wenn, dann zum Spaß. So, wie wenn ich „Alter" sage.

SZ: Sie bezeichnen Kiezdeutsch als Dialekt, also als etwas, das bleibt und dauerhaft ins Deutsche einsickern könnte.

WIESE: Das wäre schön. Wobei: nicht einsickern. Es stößt zum Hochdeutschen dazu, zu unserem bunten Spektrum.

SZ: Sie sagen: schön. Trotzdem gibt es keinen Dialekt, der einen so miesen Ruf hat. Vulgär und falsch, heißt es gern.

WIESE: Dialekte haben allgemein keinen guten Ruf in Deutschland. „Das ist meiner Mutter ihr Hut.“ Wer so etwas sagt, wird schnell abgestempelt. Beim Kiezdeutsch haben wir außerdem viele Jugendliche, die mehrsprachig sind, mit Migrationshintergrund. Auch das ist negativ besetzt. Migrationshintergrund: Das Wort wird mit Sprachproblemen assoziiert, nicht mit Innovation.[...]

SZ: Sprechen Ihre Studenten auch Kiezdeutsch?

WIESE: Viele können das. Die sind dem Jugendalter ja noch nicht lange entwachsen.

SZ: Sie zitieren in Ihrem Buch „Kiezdeutsch“ auch den „Tatian“, eine althochdeutsche Übersetzung lateinischer Evangelientexte: als Beleg dafür, dass die oftmals wilde kiezdeutsche Stellung der Verben, die im Hochdeutschen falsch ist, an sich nichts Neues ist. „Erino portun ih firchnussu“ - „Eiserne Türen ich zerschmettere."

WIESE: Schön, nicht? Bei der Verbstellung gibt uns das Kiezdeutsch Optionen zurück, die wir verloren hatten. Es passt einfach sehr gut in das System des Deutschen, und vieles, das wir in Kiezdeutsch finden, gibt es auch in anderen Varianten des Deutschen so ähnlich, zum Teil nur nicht so ausgeprägt oder so systematisch.

SZ: Zum Beispiel?
ZEIT: Was ist das Besondere an dieser Sprache?

MAROSSEK: Dieser Soziolekt mischt vor allem deutsche und türkische Wörter und Grammatikstrukturen. Er verzichtet, wie das Türkische, auf Artikel und Präpositionen: Gehst du Bus? Kommst du mit Kino? Wir gehen Disko. Und es fallen sogenannte prosodische Merkmale auf: abgehacktes Sprechen und eine kehlige, aufgeregte Sprachmelodie besonders in Situationen, in denen es ums Prestige geht.

ZEIT: Wo haben Sie geforscht?

MAROsSEK: Ich habe ein Jahr lang 30 Berliner Schulen in allen Bezirken besucht. Ich habe mich in den Klassen als Referendarin vorgestellt und einfach zugehört, Notizen gemacht, Dialoge aufgeschrieben. Die Ausbeute war großartig. Meine Arbeit hat immerhin 400 Seiten.

ZEIT: Was ist Ihnen besonders aufgefallen? MAROSSEK: Dass vieles, was aggressiv klingt, gar nicht so gemeint ist. Es wird eher als ,rituelle Beschimpfung“ verwendet.[...]

ZEIT: Was ist das Ergebnis Ihrer Dissertation?

MAROSSEK: Meine erste These: Berliner ohne Migrationshintergrund verwenden unreflektiert die türkische oder arabische Frage- und Antwortsyntax. Die zweite These: Das fällt gerade Berlinern leicht. Denn unser Dialekt hat ähnliche grammatische Strukturen. Berliner sagen auch: Bist du auf Arbeit? Ich bin mit Auto.

ZEIT: Und diese Mischsprache spricht man nicht nur in bestimmten sozialen Milieus?

MAROSSEK: Nein, auch auf dem Gymnasium in Zehlendorf. Besonders, wenn es um Prestige und Identität geht. Dann redet ein Gymnasiast gern wie der große starke Ghettojugendliche, der unangreifbar ist.

ZEIT: Machen das vor allem die Jungen?

Marossek: Nein, Mädchen sprechen ganz ähnlich. Sie sind nur ruhiger, weniger aggressiv. Sie schimpfen anders. Liebevoller.

ZEIT: Wie schimpft man denn liebevoll?

Marossek: „Schätzchen, zick jetzt mal nicht so rum.“ Ein Junge würde sagen: „Alter, ich ficke deine Mutter.“ 


\begin{abstract}
WIESE: „Ich bin Alexanderplatz.“ So etwas schnappe ich oft in der S-Bahn auf. Nominale ohne Präposition und Artikel. Das ist weit verbreitet, wenn jemand mitteilen möchte, wo er gerade ist. Ein paar Studierende von mir haben dazu mal eine schöne Hausarbeit geschrieben. Der Titel war das Zitat eines Mannes, der gerade in einer Straße in Potsdam unterwegs war und das am Handy laut mitteilte: „Ich bin Thomas Mann.“
\end{abstract}

ZEIT: Sollten auch Lehrer diesen Soziolekt beherrschen?

MAROSSEK: Wenn Lehrer Jugendcode benutzen, wirkt das uncool oder wird als herablassend empfunden. Ich habe sogar erlebt, dass Schüler einen Lehrer, der versucht hat, so zu sprechen, nicht mal verstanden haben. Die haben den korrigiert!

ZEIT: Aber können sich Lehrer dem überhaupt entziehen?

MAROSSEK: Kaum. Diese Sprache scheint sich leicht im Gehirn festzusetzen. Manchmal rutscht auch Erwachsenen so etwas heraus: „Ich geh Bäcker. Soll ich dir was mitbringen?“ Und im Lehrerzimmer habe ich gehört: „Margit, gehst du Kopierer?“

Dass die heutigen Tendenzen in der deutschen Umgangssprache nichts Exotisches und Aggressives an sich haben, sondern bereits im System des Standarddeutschen angelegt sind (durch lokale dialektale Einflüsse [Berlinisch] wie die Erstsprachen Migrantenjugendlicher geprägt sind und die Form ,ritueller Beschimpfungen ' unter jungen Menschen annehmen), bringt die beiden Aussagetypen auf einen gemeinsamen Nenner. Die Linguistinnen sind sich darüber hinaus auch darin einig, dass die ,Kiez- / Kurz-Sprache‘ von Jugendlichen mit und ohne Migrationshintergrund gleichermaßen verwendet wird und zu ihren Leitmerkmalen die Artikel- und Präpositionstilgung in lokalen präpositionalen Phrasen gehört. Während WIESE den Gebrauch des ,Kiezdeutschen` auf junge Sprecher im Alter zwischen 12 und 20 Jahren einschränkt, spricht MAROSSEK Lehrkräften die Kompetenzen im ,Kurzdeutschen“ nicht ab - dies aber mit dem Verweis darauf, dass es von ihren Schutzbefohlenen als ,herablassend ‘ und höchstwahrscheinlich ,sozial inkompetent' empfunden wird, was sich in ihrer sofortigen Bereitschaft zur Korrektur niederschlägt.

\section{Schluss}

Der vorliegende Beitrag geht der medialen Berichterstattung zum ethnolektalen Sprechstil nach. Ein besonderes Augenmerk richtet sich hier auf hochaktuelle Veröffentlichungen deutscher SprachwissenschaftlerInnen, die somit Resultate ihrer im Rahmen der sprachorientierten Projekte durchgeführten Fallstudien anschaulich machen. Es werden hier linguistische sowie journalistische Standpunkte zum Wandel der deutschen Sprache enthüllt und kommentiert, und zwar mit dem Ziel, den aktuellen Wissensstand über Kreuzungs- 
und Mischungsstrategien in jugendlich dominierten Kreisen Deutschlands zu beleuchten und dafür zu sensibilisieren. Für diese klar festgelegte Aufgabe haben sich Medienbeiträge als besonders dankbares Material erwiesen.

\section{Literatur}

Aslan, Sema (2005): Aspekte des kommunikativen Stils einer Gruppe weltläufiger MigrantInnen türkischer Herkunft: die „Europatürken“. In: Deutsche Sprache 32 (4):327-356.

Auer, Peter (2003): „Türkenslang“ - ein jugendsprachlicher Ethnolekt des Deutschen und seine Transformationen. In: HACKI-BuHOFER, ANNELIES (ed.): Spracherwerb und Lebensalter. Tübingen / Basel, 255-264.

AUER, PETER: Jugendsprache in Stuttgart unter dem Einfluss von kultureller, ethnischer und sprachlicher Vielfalt: http://www.germanistik.unifreiburg.de/auer/?Projekte:Laufende_Projekte:Jugendsprache_in_Stuttgart_unter_dem_Einfluss_von_kultureller\%2C_ethnischer_und_sprachlicher_Vielfalt (23.05.2005).

BAX, DANIEL: Abschwellender Straßenslang. Zwischen Fluchen und Schnöseln: KanakSprak ist Popliteratur geworden. Doch was wird aus deren Bannerträgern, wenn auch diese Mode verebbt?: http://www.taz.de/1/archiv/print-archiv/printressorts/digi-arti$\mathrm{kel} /$ ?ressort=li\&dig=2001/10/10/a0216\&cHash=e4aae444fc (10.10.2001).

Braun, Michael: Was Problem. Der Kauderwelsch-Kult: Kanak Sprak wird Alltags Sprak: https://www.freitag.de/autoren/der-freitag/was-problem (26.05.2000).

CANoĞLu, Hatice Deniz (2012): Kanak Sprak versus Kiezdeutsch - Sprachverfall oder sprachlicher Spezialfall? Eine ethnolinguistische Untersuchung. Berlin.

CINDARK, IBRAHIM (2005): „Die Unmündigen“. Eine Fallstudie der emanzipatorischen Migranten. In: Deutsche Sprache, 32 (4):299-326.

CindaRK, IBRAHIM (2010): Migration, Sprache und Rassismus. Der kommunikative So-zialstil der Mannheimer „Unmündigen“ als Fallstudie für die „emanzipatorischen Migranten“. Tübingen.

Clyne, Michael (2000): Lingua Franca and Ethnolects in Europe and Beyond. In: Sociolinguistica 14:83-89.

DiRIM, ĬNCI / AUER, PETER (2004): Türkisch sprechen nicht nur die Türken. Über die Unschärfebeziehung zwischen Sprache und Ethnie in Deutschland. Berlin / New York.

HARTwIG, GunTHER: Auch Lehrer sprechen „Kiezdeutsch“: http://www.moz.de/artikel-ansicht/dg/0/1/1310640 (12.08.2014).

HaRUnA, Hadija: Weissu - is krasse Sprache! Jugendliche Migranten mischen das Hochdeutsch auf: http://www.fluter.de/de/sprachen/heft/9536/ (30.08.2011).

Heine, MatTHIAs: Kurzdeutsch: „Ich gehe Döner“ ist längst im Sprachgebrauch angekommen: http://www.welt.de/kultur/literarischewelt/article151650918/Ich-gehe-Doener-ist-laengst-im-Sprachgebrauch-angekommen.html (30.01.2016). 
Heine, Matthias: Streit um Sprache: In Wahrheit ist Kiezdeutsch rassistisch http://www.welt.de/kultur/article129622721/In-Wahrheit-ist-Kiezdeutsch-rassistisch.html (30.06.2014).

HiNRICHS, UwE (2012): Hab isch gesehen mein Kumpel. Wie die Migration die deutsche Sprache verändert. In: Der Spiegel 7/2012, 104-105.

HinRICHS, UwE (2013): Multi Kulti Deutsch. Wie Migration die deutsche Sprache verändert. München.

Hymes, Dell (1979): Zur Ethnographie der Kommunikation. Frankfurt am Main.

KAllMEYER, WeRnER (Hg.) (1994): Kommunikation in der Stadt. Teil 1: Exemplarische Analyse des Sprachverhaltens in Mannheim. (= Schriften des Instituts für deutsche Sprache 4.1). Berlin / New York.

Kallmeyer, Werner (Hg.) (1995): Kommunikation in der Stadt. Teil 2: Ethnographien von Mannheimer Stadtteilen. (= Schriften des Instituts für deutsche Sprache 4.2). Berlin / New York.

KEIM, INKEN (1995): Kommunikation in der Stadt. Teil 3: Kommunikative Stilistik einer sozialen Welt „kleiner Leute“ in der Mannheimer Innenstadt. (= Schriften des Instituts für deutsche Sprache 4.3). Berlin / New York.

KEIM, INKEN (1997): Formelhaftes Sprechen als konstitutives Merkmal sozialen Sprechens. In: Selting, Margret / SANDig, BARBARA (Hg.): Sprech- und Gesprächsstile. Berlin / New York, 318-344.

KeIM, InKen (2008): Die „türkischen Powergirls“. Lebenswelt und kommunikativer Stil einer Migrantinnengruppe in Mannheim. Tübingen.

KERN, FRIEDERIKE / SELTING, MARGRET (2006): Einheitenkonstruktion im Türkendeutschen: Grammatische und prosodische Aspekte. (= Zeitschrift für Sprachwissenschaft 25), 239-272.

Koldehoff, Stefan: „Das Deutsch der Migranten gibt es nicht.“ Die Soziolinguistin Inken Keim über Standard- und „Kiezdeutsch“: http://www.deutschlandfunk.de/dasdeutsch-der-migranten-gibt-es-nicht.691.de.html?dram:article_id=56817 (13.03.2012).

KRISCHKE, WOLFGANG: Kiezdeutsch: »Ischwör, morgen bin ich Arzt!«: http://www.faz.net/aktuell/feuilleton/buecher/rezensionen/sachbuch/kiezdeutsch-untersucht-von-soziolinguistin-diana-marossek-14167926.html (14.04.2016).

KRISCHKE, WoLFGANG: Sprache: »Ich geh Schule«: http://www.zeit.de/2006/27/CKiezdeutsch (29.01.2006).

KrischKe, WolfGang: Uwe Hinrichs: Multi Kulti Deutsch. Das ist mehr einfach wie alter Mann Schoppenhauer: http://www.faz.net/aktuell/feuilleton/buecher/rezensionen/sachbuch/uwe-hinrichs-multi-kulti-deutsch-das-ist-mehr-einfach-wie-alter-mannschopenhauer-12678983.html (24.11.2013).

KRUMMHEUER, BIRGIT: Wie Jugendliche reden: Türkendeutsch als Szenesprache: http://www.rp-online.de/kultur/kunst/tuerkendeutsch-als-szenesprache-aid-1.2033555 (20.03.2007). 
LiNKO, HeIKE: Sprache im Wandel: Präpositionen überbewertet: http://www.faz.net/ aktuell/gesellschaft/sprache-im-wandel-praeposition-ueberbewertet-13016742.html (29.06.2014).

LÜBER, KLAUS: Kanak Sprak: An sozialen Brennpunkten verändert sich auch die deutsche Sprache: http://www.welt.de/print-welt/article208701/Kanak-Sprak.html (5.04.2006).

MarosseK, Diana (2016): Kommst du Bahnhof oder hast du Auto? Warum wir reden, wie wir neuerdings reden. Berlin.

SchrAmm, MARTin: „Kanak Sprak“: Verhunztes Deutsch oder Sprachkultur?: http://www.br.de/radio/bayern2/wissen/iq-wissenschaft-und-forschung/gesellschaft/Sprachverfall100.html (31.05.2011).

Schwitalla, Jochannes (1995): Kommunikation in der Stadt. Teil 4: Kommunikative Stilistik zweier sozialer Welten in Mannheim-Vogelstang. (= Schriften des Instituts für deutsche Sprache 4.4). Berlin / New York.

SERRAO, MARC Felix: Jugenddialekt Kiezdeutsch: „Ich bin Alexanderplatz“: http://www.sueddeutsche.de/leben/jugenddialekt-kiezdeutsch-ich-bin-alexanderplatz1.1278128 (8.02.2012).

STRASSMANN, BURKHARD: Herr Lehrer geht Kopierer. Eine Soziolinguistin erforscht, wie sich Berliner Schüler Türkisch aneignen: http://www.zeit.de/2014/27/soziolinguistin-diana-marossek-deutsch-tuerkisch (10.07.2014).

STUTE, DenNIS: Sprache: Zukunftssprache Kanak Sprak?: http://www.dw.com/de/zukunftssprache-kanak-sprak/a-15208590 (04.07.2011).

TwIEHAUS, JENS: Jugendsprache: Lass ma' lesen, yallah!: http://www.spiegel.de/schulspiegel/leben/jugendsprache-lass-ma-lesen-yallah-a-811877.html (28.01.2012).

Von LeszCZynski, UlRIKE: Sprachwandel: „Gehst du Bus?“ Kiezdeutsch gibt's bald ohne Kiez: http://www.welt.de/vermischtes/article129589372/Gehst-du-Bus-Kiezdeutsch-gibts-bald-ohne-Kiez.html (29.06.2014).

WIESE, HEIKE: Kiezdeutsch - ein neuer Dialekt: http://www.bpb.de/apuz/32957/kiezdeutsch-ein-neuer-dialekt (16.02.2010).

WIESE, HeIKE (2012): Kiezdeutsch. Ein neuer Dialekt entsteht. München. 